\title{
Next Generation Genetic Counseling: Introduction to the Special Issue
}

\author{
Myra I. Roche • Christina G. S. Palmer
}

Received: 21 April 2014 / Accepted: 1 May 2014 / Published online: 18 May 2014

(C) National Society of Genetic Counselors, Inc. 2014

The genesis of the idea for this special issue, as well as its title, "Next Generation Genetic Counseling," originated in January 2012. As the newly hired project manager for the University of North Carolina-Chapel Hill's Clinical Sequencing Exploratory Research (CSER) consortium project, NCGENES, Myra had begun speculating just how the adaptation of Next Generation Sequencing (NGS) would affect genetic counseling practice (Roche 2012). Later that spring, at the American College of Medical Genetics meeting, the message that NGS would have an unprecedented impact on genetic counseling was delivered loudly and clearly but it seemed that too few of the voices sending that message belonged to genetic counselors.

The idea behind this Special Issue is simply this: tap into the collective wisdom of the genetic counseling community to explore how NGS's clinical application would impact current genetic counseling practices and ponder future implications by providing a platform on which the voices of genetic counselors could be heard. Since it was still early in the adaptation phase, we suspected that few empirical studies analyzing NGS and genetic counseling would be completed in time for our submission deadline. Therefore, we decided to cast a wide net in order to capture a broader swath of the early adaptation experiences of genetic counselors as they transitioned from traditional approaches in testing, counseling, and training to what promised to be an exciting, if uncertain, future. We

\section{I. Roche ( $\square)$}

Department of Pediatrics and Genetics, Center for Genomics and Society, University of North Carolina, Chapel Hill, NC, USA e-mail: myra_roche@med.unc.edu

\section{G. S. Palmer}

Department of Psychiatry \& Biobehavioral Sciences, UCLA Department of Human Genetics, UCLA Institute for Society \& Genetics, University of California-Los Angeles, Los Angeles, CA, USA challenged our colleagues to think, discuss, and write about what they saw as the critical emerging issues facing our profession. We wanted to "crowd source" their ideas and hear about strategies to combine the essence of what our profession has always contributed with new, best practices for the future as we moved together towards Next Generation Genetic Counseling (NGGC).

Our call for papers invited submissions describing the evolution of genetic counseling and education strategies relevant to whole genome or whole exome sequencing; counseling and ELSI issues specifically related to return of diagnostic and incidental results; and the role of the genetic counselor on multidisciplinary teams offering this testing. The diverse submissions in this issue successfully answered our call. They range from empirical studies analyzing such bread and butter topics as informed consent and return of results to case studies describing how practicing genetic counselors are grappling with these same issues in their clinics. Throughout, we have kept the focus on the genetic counselors themselves as they adapt to expanded niches and actively create new ones.

In response to the initial concern that this call for papers might be a bit premature and thus might receive a muted response, this hefty issue delivers a resounding reply squashing any doubts that NGGC has become a hot topic in the genetic counseling community. We are pleased that so many genetic counselors and their colleague have answered this call. This completed issue includes an astonishing number of papers, 28 in all, representing both US and Canada. It includes 14 original research studies, ten professional development pieces, two case studies, one literature review and a book review. Submissions span both clinical and research populations, studying those that are healthy and those that are not, and they peer into the future though multiple lenses including those of practitioners, students, and program directors. 
Although the effects predicted by the adoption of genomic medicine have been likened to a tsunami, setting aside the hyperbole for a moment, how can we best measure and describe the magnitude of changes that will be required to adapt genetic counseling practice? Are the set of practices needed for successful NGGC so qualitatively different that a fundamental rebooting should be done, or are the bedrock issues sound enough that this newest technology can, once again, be incorporated with only a minimum amount of fuss? In the lead off article, Hooker and colleagues argue the predicted impact of this technology is unprecedented and they foresee far-ranging ripples that will touch our core counseling skills in areas such as facilitating informed consent, managing patient expectations, and tailoring results. In the second article, Wicklund and Trepanier choose, instead, to emphasize the soundness of our core skills, confident that these will enable us to navigate through the upcoming Class III rapids, but only if we also increase our dexterity and maneuverability. They foresee several threatening eddies along the way; namely, managing the increased volume of test results, developing new, reimbursable service delivery models, expanding the workforce, and ensuring an adequate foundation of outcomes research upon which to base our practice.

One looming iceberg, already sighted by many as a formidable barrier blocking the smooth integration of NGS into clinical practice, is the massive effort required for variant analysis and interpretation that has, thus far, remained stubbornly resistant to a high throughput pace. Facio, Lee, and O'Daniel explore the largely uncharted territory surrounding current NGS technologies as they illustrate the clinical applications and predict their impact on the practice of genetic counseling. Their guide is particularly useful for genetic counselors who are beginning to populate the specialized niche of the molecular analyst but also serves as a useful reminder for everyone else that deeper knowledge of genomic sequencing will be essential for the competent practitioner of NGGC.

We then turn to examine key issues that arise as genetic counseling practice transitions from experiences with genomic tests such as microarrays to clinical genomic sequencing. We open this section with a review article by Lohn et al. who examine one of the most contentious topics in clinical genomics; the return of incidental findings. Defined as "discoveries made as a result of genetic testing that are unrelated to the indication for the test," the authors conclude that, although a consensus mandating the return of analytically valid and medically actionable incidental findings has begun to form, criteria about which types should be returned, and how to address the accompanying logistical and counseling issues, are still very much in flux. Their call for empirical studies to help guide these difficult decisions is answered, in part, in the next article by Reiff et al. in their study of health care providers. These voices capture the double-edged sword nature of the Faustian bargain we strike when we eagerly seize genomic testing's promise of increased diagnostic capability while blithely trying to ignore the growing millstone of obtaining informed consent for an increasing range of incidental findings. And though the scope of incidental findings grows with each technological advance, the case studies by Helm et al. gently remind us that one of the most fraught-laden pieces of genetic information is, incidentally, the type that could be revealed by very early techniques: the attribution of paternity. Their experiences of disclosing chromosomal microarray results consistent with first degree parental consanguinity is instructive both in what it tells us about families expectations about the true scope of potential results that genomic testing can reveal but also, sadly, about our often limited ability to adequately prepare them for this information.

Machini and colleagues then whisk us away from the intimacy of the clinic to survey genetic counseling practice from $50,000 \mathrm{ft}$ up. They examine ways in which clinical sequencing can be expected to impact our service delivery system and note several barriers to clinical adaptation. These obstacles range from a painfully familiar one (billing and reimbursement) to speculation about the degree to which our limited workforce capacity is exacerbated by our reliance on service delivery models that do not scale. In their calls for student and practitioner training, they also worry about the meager expertise in variant analysis and interpretation. Nardini et al. push the settings envelope one step further, to our next frontier; newborn screening. The authors optimistically conclude that, although experience with clinical sequencing in this setting has been limited, thus far, genetic counselors' experience with sequencing in other settings should pave the way for a smoother learning curve when rolled out in the newborn setting. We then conclude with two analyses of genetic counseling practice. At the micro level, Williams et al. present a time analysis of one step in the process: that of acquiring and reviewing participants' medical records prior to sequencing. Their estimates, an average of four months per participant and ranging between three and nine months, should surprise no one who has ever requested medical records in the US. As genomic sequencing intercalates itself into clinical practice, the relative value of this traditional approach will merit a re-evaluation, especially if sequencing costs precipitously decline. In contrast, Radford et al. focus on macro level issues and predict that access to services, insufficient reimbursement, and the threat of genetic discrimination will only worsen as demand for genetic testing grows. They propose that genetic counselors position themselves as information hubs and extend dendritically out to form community partnerships in order to magnify the limited availability of genetic expertise.

Returning again to the high profile issue of establishing criteria for the return of incidental results, we next hear from both professionals and patients about how these decisions should be guided. For the professionals, the devil is indeed 
in the details. Asked by Grove and colleagues to "bin" or categorize several gene-variant pairs, professionals struggled to make consistent decisions about which results would merit obligatory return vs. those that would not. Although the study was completed before the American College of Medical Genetics and Genomics recommendations were released (Green et al. 2013), it confirms that criteria have evolved with clinical adaptation and that lack of unanimity amongst professionals is the rule rather than the exception. In developing evidence-based professional guidelines to help establish standards of care, we may need to re-define processes such as informed consent and result disclosure as events that occur over time and over the lifespan of the patient.

Although professionals may be conflicted, patients, apparently, are not. Hitch et al. found that their Lynch syndrome patients, all who had received uninformative genetic testing results by traditional testing methods, held nearly unanimous preferences as to which types of genomic sequencing results should be returned: as one patient so colorfully phrased it, "Bring it on!" Levenseller et al. queried both professionals and families and identified similar discrepancies as professionals expressed much more hesitancy about the return of some findings. In an interesting twist, professionals aligned themselves more closely with the adolescents than the parents in this study believing that teens should be actively involved in decisions about disclosures pertaining to them. The parents surveyed by Erickson et al. likewise, took a similarly protective stance when asked about their interest in a hypothetical test that could tell them with 20-90\% accuracy the chance that their child would develop a mood disorder. While favoring testing if results were highly predictive, most responded that they would not communicate the results to the child at all $(17 \%)$, until symptoms began (17\%) or until the "child" turned $18(25 \%)$. Studies addressing if, when, and how parents communicate results to their children are sorely needed to determine if this will be one of those rare cases where hypothetical intention matches actual behavior.

Even with wide open communication lines it can be instructive to learn which parts of a message are more likely to be garbled especially when relying on information delivery models that deviate from the traditional face-to-face encounter with a genetics professional. Schmindlen et al. uncovered evidence of several misunderstandings in their retrospective study of an on-line personalized medicine site that analyzed queries posted to the genetic counselors. Although only a small proportion of the tested population posed questions, those that did had trouble distinguishing between absolute and relative risks and showed a tendency to over-estimate the predictive power of the results. The understandings of those who did not post questions in this study remain unknown but these data would be useful in evaluating an on-line approach. Circling back to the clinic, we learn about a case described by Huang et al. that illustrates both the tantalizing potential and the messy realities of sequencing and interpreting multiple genes simultaneously. They identified potentially causative variants in, not one, but two genes in a patient with cone-rod dystrophy. As is true for many such variants, however, the avenues by which pathogenicity could be assessed were limited leaving the patient with ambiguous results and unclear recommendations. This predicament, uncomfortable as it is for both patient and counselor, will likely become more common as our skills in variant interpretation continue to lag far behind those in variant identification.

The successful adoption of a new technology can depend upon the clinical setting and, as Machini et al. observed in their study, genomic sequencing was more likely to be ordered in a pediatric setting than in an oncology or a prenatal one. One reason for this discrepancy could stem from the traditions and goals of a practice within a discipline. In pediatrics, the primary goal is to identify the etiology of a condition and so diagnostic technologies, such as genome sequencing, are more readily accepted irrespective of their often-limited clinical utility. There also seems to be a higher tolerance for ambiguous results, as many more variants are reported than can ever be satisfactorily adjudicated. Casting such a wide net is also indulged because our knowledge of the spectrum of genetic variants associated with disruptions of growth and development is still woefully under-developed. However, in disciplines whose primary purpose has been treatment, such as oncology, historically, only tests with robust clinical utility have gained traction, been translated into practice guidelines, and have shaped the training and practice of genetic counselors.

With these distinct disciplinary traditions serving as a background, the next series of articles describe the genetic counseling implications of the rapid clinical adoption of gene panels. Gene panels can be regarded as occupying a middle ground between the one-gene-at-a-time approach and comprehensive genomic sequencing. Ideally, they target specific sets of genes/mutations with established clinical relevance to a phenotype. In the best of all possible worlds, panels reap the bounty of increasing diagnostic sensitivity yet manage to sidestep much of the cost in interpretation and return of incidental findings commonly encountered in a whole genome approach. Just as our experiences with microarrays have illuminated what to expect on the clinical path to genomic sequencing, experiences with panels can also be instructive as we calculate at which point increasing the number of genes on a panel meets the point of diminishing clinical returns.

Platt et al. ignite this series of articles with an eye-opening look at how the composition of large mitochondrial gene panels can translate into unsuspected and undesired clinical dilemmas to nearly the same degree as occurs using a whole genome approach. They examined panels, ranging in size from 100 to 1024 genes, offered by seven US laboratories. As might be expected, there was a direct relationship between 
the size of the panel and the number of genes whose analysis would be of limited, or no, clinical diagnostic use. Arguably worse, panels were constructed that could return incidental findings that neither the counselor nor the patient were expecting including results that predicted substantial risks for cancer and for untreatable, adult-onset, neurological disorders. Their analysis of the corresponding consent forms showed little or no mention of these worrisome downstream risks; only one mentioned the possibility of incidental findings and none provided a mechanism to allow patients to opt out of learning information. The authors recommended that clinicians, including genetic counselors, take responsibility for examining the contents of panels and encouraged clearer communication by the laboratories about the spectrum of results that could be returned.

Hiraki et al. then review the characteristics that make a gene a good candidate for inclusion on a panel for assessing the risk of breast, ovarian and colon cancers. Unlike those of mitochondrial panels, designers of cancer panels have greatly benefitted from a broad consensus as to which genes should be included. The authors focus on panels that assess individuals who are at high or moderately increased risk and caution that pedigree analysis alone fails to identify some who are at significant risk. Due to its increased sensitivity, cancer panel testing can extend genetic risk assessment to a broader population and can provide information that helps clinicians and patients weigh the pros and cons of various interventions. But the genetic heterogeneity that makes panel testing so attractive comes at an exacting, albeit familiar, cost; more variants of uncertain clinical significance are identified, multiple potentially causative mutations may be found, and pleiotropic mutations confer risks for different cancers, some of which are amenable to effective treatment while others are not.

Cancer genetic counselors have been navigating the transition from a practice guideline, single gene testing approach to one based on the broader use of cancer gene panels even as surveillance and management recommendations fluctuate. Lundy et al. measured the level of congruence between cancer genetic counselors' evaluation of prototypical cancer pedigrees with recommendations from the National Comprehensive Cancer Network (NCCN). Although counselors demonstrated high concordance between their choice of a single gene test and NCCN guidelines, they were more likely to consider cancer panel use for highly penetrant cases that lacked defining features of a particular syndrome, even in the absence of current practice guidelines to support this decision.

While impatiently waiting for new practice guidelines to emerge, patients must still be seen and counseled. One pressing issue becomes how to best obtain informed consent given the heterogeneity of the genes on a typical cancer panel and the limited time available during a session. In an attempt to streamline the informed consent and result disclosure processes, Fecteau et al. propose a model of "gene stratification" to categorize two critical parameters of identified mutations: the degree of risk conferred and the degree to which management guidelines exist. Keeping one step ahead of the patient in this rapidly changing environment can be difficult for the individual counselor. By adopting a "crowd source" method, a group of Colorado counselors, described by Schneider et al., formed a working group to collectively improve their understandings of the evolving panel compositions, assess their clinical utility, and help define appropriate candidates for testing. This collaborative process employed a divide-and-conquer methodological approach by which genes were assigned to individuals for review and standardized summary and then later discussed during monthly conference calls.

As Platt and colleagues convincingly demonstrated in their study, neglecting to establish a stringent chain of responsibility to define the risks and benefits and potential downstream effects of testing is harmful to patient care. Laboratories offering genomic sequencing have developed more robust consent forms over time but these continue to evolve as more experience is gained. And as new methods are developed to support flexible patient decisions about result disclosure, the responsibilities of what constitutes adequate informed consent, and how that can best be provided, will shift again. Genetic counselors have unique, clinically-honed insights that can enhance the responsible deployment of laboratory tests by predicting which downstream effects demand incorporation into pre-test counseling and education, thereby helping patients make complex decisions about return of results.

It should come as no surprise, then, that as clinical testing laboratories have proliferated, they have become incubators of one of the fastest growing niches for genetic counselors. Swanson et al. describe the roles and responsibilities in this burgeoning environment and predict that the counselors in these positions will help leverage the limited amount of genetic expertise as the demand for testing grows. Sharing the common currency of core counseling skills with their clinical counterparts, the difference between them lies primarily in their educational targets.

The increase in the number of clinical genomic research projects, such as the Michigan Oncology Sequencing Project described by Everett et al. has spawned the development of the second fastest-growing niche for genetic counselors. As in Michigan, genetic counselors across the country are adopting numerous roles within such projects ranging from project coordination to acting as molecular analysts in interpreting variants, in addition to the more traditional roles of obtaining consent and disclosing results.

Egalite and colleagues provide evidence that the role for genetic counselors in traditional research settings is also ripe for expansion. Ironically, though the debate about the appropriate return of results began in the research arena, the offer 
and acceptance of genetic counseling in many projects remain haphazard, even when clinically significant results are generated. The authors' content analysis of informed consent templates and guidelines by Canadian institutional review boards showed marked variability in how these documents addressed the return of results and when and how genetic counseling would be made available to participants. They concluded that improving the clarity and consistency of these documents would promote the informed consent process and could result in genetic counseling being provided to more participants.

In the papers reviewed thus far, we have seen a snapshot of NGGC practice in its infancy, circa 2013. Peering into the future, we might just barely discern a glimmer of which changes lie in the near future. But what might NGGC look like, say, in the year 2017? The quickly shifting technologies may mock our attempt at a 4-year projection as a foolhardy enterprise. But we can capture one slice of the future by studying current genetic student training and cataloging if, when, and how genomic topics have wound their way into the curricula. Noss and colleagues surveyed genetic counselors who graduated between 2008 and 2011 to document how personalized genetic testing, defined broadly as "genome-based testing to assess a person's risk, or susceptibility, of developing a disorder with either a known or suspected genetic component" was taught during that time. They found a statistically significant increase in the amount of content about whole genome sequencing, Genome Wide Association Studies and Direct-to-Consumer testing. In retrospect, their respondents felt that a greater emphasis on clinical utilization, including test interpretation and identifying appropriate testing candidates, would have been even more valuable. Profato and colleagues asked a similar question to those on the other side of the desk; the program directors. Nearly all the respondents agreed that genomic medicine was steadily becoming assimilated into program curricula, spurred even more by its inclusion on the recent Practice Based Competencies. The number of content hours devoted to this topic ranged from between 2 to $35+$ hours and adoption of this content would have been even speedier had it not been hindered by limited access to content experts and to published clinical experiences. We hope the papers in this issue will begin to fill that void.

How did program directors see genetic counselor roles in the future? Some believed they would be recognizably similar to current ones while others predicted a steady migration towards other settings such as primary care or to other sub-specialty settings such as cardiology and neurology. Most agreed, however, that, regardless of the setting, the core skills would predominate; the ability to explain genetic technologies, interpret data and translate it into clinically meaningful information, communicate uncertainty and, perhaps the biggest challenge for the future, recognize how much and which information to share with patients.

The final two futuristic views stem from a common belief in the influence of the same seminal landmark; personalized medicine. In the first, Mills and Haga predict that the general model of genetic counseling will be preserved but foresee marked changes to its delivery system. These changes, spurred by the predominance of post-test encounters and the changing types of diseases for which testing will occur, could transform the nature of genetic counselors' expertise from an emphasis on those factors that induce risks to those that reduce risk. The delivery of this expertise, in turn, would necessitate a more directive stance as counselors advise generally healthy people about disease screening protocols and life style changes.

Gutkind and Kennedy, in their creative non-fiction book, An immense new power to heal: The promise of personalized medicine, reviewed in this issue by Liz Varga, oscillate between whether the magnitude of changes ahead are better classified as "revolutionary" or "evolutionary." Perhaps looking back with the hindsight of the next 45 years, we might conclude that the model of punctuated equilibrium, as first described by Gould and Eldredge (1977), serves as a better metaphor when we are finally able to step back and take stock of which elements have remained static amidst the current clamor of change.

Finally, we wish to graciously acknowledge the virtual village that we called upon to translate our vision of this special issue into a reality. First, we wish to thank all of the authors who submitted manuscripts. We see this issue as a beginning and truly hope it inspires colleagues to write about their experiences with NGGC to insure that genetic counselor voices are heard. Secondly, we would like to acknowledge all of the reviewers (numbering at least 60 !) who donated their time and expertise to enhance the ultimate quality of this issue. Many thanks also to Bonnie LeRoy (Editor-in-Chief), Patricia McCarthyVeach (Assistant Editor), and the editorial board members for their continued help and support.

We hope the manuscripts in this issue will provide food for thought, discussion, and debate and inspire students and genetic counselors to investigate, analyze and discover how we can best move together towards next generation genetic counseling.

Acknowledgments Ms. Roche is supported by the National Human Genome Research Institute of the National Institutes of Health, Grants Number P50HG004488 and U01HG006487 with additional support from the UNC Cancer Research Fund and the UNC Lineberger Comprehensive Cancer Center.

Conflict of interests Myra I. Roche and Christina G.S. Palmer declare that they have no conflict of interests. 


\section{References}

Green, R. C., Berg, J. S., Grody, W. W., Kalia, S. S., Korf, B. R., Martin, C. L., et al. (2013). ACMG recommendations for reporting of incidental findings in clinical exome and genome sequencing,
American college of medical genetics. Genetics in Medicine, 15, 565-574. doi:10.1038/gim.2013.73.

Gould, S. J., \& Eldredge, N. (1977). Punctuated equilibria: the tempo and mode of evolution reconsidered. Paleobiology, 3(2), 115-151.

Roche, M. I. (2012). Moving toward nextgenetic counseling. Genetics in Medicine. doi:10.1038/gim.2012.84. 